\title{
A model for optical/X-ray correlation in black hole $\mathrm{X}$-ray binaries
}

\author{
Alexandra Veledina* \\ University of Oulu \\ E-mail: Glexandra.veledinadoulu.fi] \\ Juri Poutanen \\ University of Oulu \\ E-mail: Guri.poutanendouLu.fi \\ Indrek Vurm \\ Racah Institute of Physics, Hebrew University of Jerusalem
}

Simultaneous optical and X-ray observations of black holes in X-ray binaries (GX 339-4, XTE J1118+480, SWIFT J1753.5-0127) show complicated shape of the cross-correlation function (CCF), with an anti-correlation preceding positive correlation. Similar CCFs were found in two neutron star X-ray binaries, Sco X-1 and Cyg X-2. We propose a novel model capable of explaining timing properties of these objects. We argue that the X-ray emission is produced by synchrotron self-Compton mechanism, which also contributes to the optical luminosity. Small variations in the accretion rate cause changes of parameters of the flow (optical depth, total luminosity), leading to variations in X-rays and optical luminosities. By simulating spectra for specific parameters, we show that fluxes in the two bands are anti-correlated. Additional contribution to the optics comes from reprocessing of the $\mathrm{X}$-rays by the outer regions of the accretion disk. We find that our model is consistent with the CCFs recently observed in Galactic black holes. From the presence of the dips in the CCFs, we can estimate the size of the synchrotron-emitting region, and from the relative amplitude of the dips to the peaks we constrain the contribution of synchrotron radiation in the optical energy band.

Fast $X$-ray timing and spectroscopy at extreme count rates

February 7-11, 2011

Champéry, Switzerland

\footnotetext{
* Speaker.
} 


\section{Introduction}

Physical processes giving rise to optical spectra of accreting black holes in low-mass X-ray binaries (LMXBs) are currently widely discussed. The contribution from the companion star in such systems usually is too faint, and the optical spectra most likely are connected to the accretion process onto the compact object and the X-ray radiation. To understand the origin of the optics,

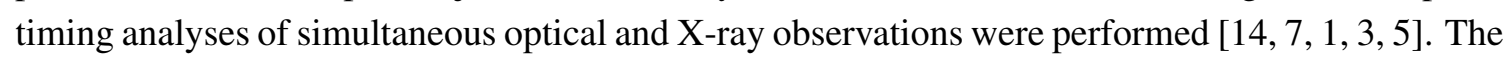
computed optical/X-ray cross-correlation functions (CCF) revealed a complicated structure with a dip in the optical light curve, preceding the X-ray peak (the so-called precognition dip), together with an optical peak lagging the X-rays. The observed behavior cannot be explained by a simple model with the optical radiation being produced by the reprocessed X-ray emission (as was shown for UV/X-ray CCF, [ [ $]$ ]). Further analysis showed that the autocorrelation function (ACF) of the optics is narrower, than that of the $\mathrm{X}$-rays [ [ $\mathrm{Z}, \mathbf{6}, \mathbf{D}]$. A number of possible mechanisms, producing such structure of the CCF, were proposed (see the discussion section in [B] $]$ and references therein). So far, the detailed calculations were made only in the model of Malzac et al. [W], where both Xray and optical emission are fed from the same magnetic energy reservoir. The model qualitatively describes the CCF and ACF of XTE J1118+480, however, with the new data on Swift J1753.50127, the applicability of the model was put under question. Also, it is not clear, whether such magnetic reservoir can be maintained.

We propose a novel model, where the optical emission is a composition of two components. The first one is coming from the synchrotron-emitting particles in the inner hot flow and is anticorrelated with the X-ray emission. The second one is originating from reprocessing of the X-rays by the outer parts of the cold accretion disk. This component is (positively) correlated and delayed relatively to X-rays. This explains both the precognition dip and the delayed peak of CCF. The model explains the entire optical to X-ray spectrum of the BHBs as well as qualitatively describes timing properties.

\section{Spectral modeling}

The X-ray spectra of Galactic black holes in their hard state are well described by Comptonization on thermal electrons [ [15, 2]], however MeV tails detected in many sources suggest the presence of non-thermal particle population in addition to the mostly thermal distribution [ए2]. These electrons contribute both to the high-energy part of the spectrum via Compton scattering and to the low-energy part by synchrotron radiation. Even a tiny fraction of non-thermal electrons dramatically increases synchrotron luminosity [20] and thus makes it a plausible source of seed photons for Comptonization. Investigations show that typical X-ray spectra of both hard- and soft-state BHBs can be modeled in terms of synchrotron self-Compton (SSC) mechanism in hybrid (thermal plus non-thermal) plasmas [ㅁ, 매, ㅁ] ].

We are modeling the emitting spectra of a hard-state accreting black hole. While the highenergy part of the spectrum is dominated by upscattered photons, the low-energy part is determined by synchrotron emission, which can fall to the optical band. The calculations are made in one-zone spherical geometry, homogeneously filled with photons and particles having isotropic distributions. The region corresponds to a hot flow in the vicinity of the black hole, which size is limited by the in- 

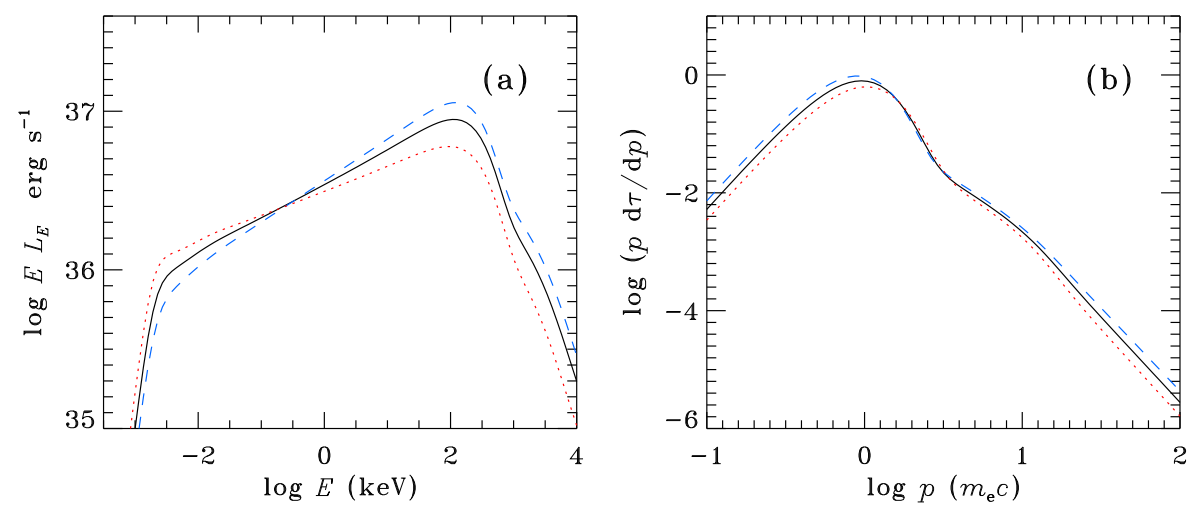

Figure 1: Simulated spectra (a) and electron distributions (b) for fiducial parameters (solid line), for increased by $20 \%$ mass accretion rate (blue dashed line) and for decreased by $20 \%$ accretion rate (red dotted line).

ner radius of (truncated) accretion disk. Particle heating results from magnetic reconnection, shock acceleration etc., typically leading to power-law distributions. The main mechanisms, responsible for particle cooling and formation of the spectra are synchrotron radiation and Compton scattering. To model the emitting spectra, which are self-consistent with their electron distributions, we use the code developed by Vurm \& Poutanen [एव].

We consider typical for hard-state BHBs parameters: the Thomson optical depth $\tau=1.0$, the magnetic field $B=3 \times 10^{5} \mathrm{G}$ and the total luminosity of the region $L=10^{37} \mathrm{erg} \mathrm{s}^{-1}$. The selfabsorbed frequency of synchrotron radiation strongly depends on the size of the source. The optically thin synchrotron emission falls into the optical energy bands $(1-10 \mathrm{eV})$, when region size is about $R \sim 10^{8} \mathrm{~cm}\left(\sim 30\right.$ Schwarzschild radii for a $10 \mathrm{M}_{\odot}$ black hole $)$. The resulting spectrum and electron distribution is shown on Fig. $\mathbf{\square}$ (solid line).

Variations in mass accretion rate $\dot{m}$ lead to changes in parameters of the system. We consider change of the total luminosity $L \propto \dot{m}$, the optical depth $\tau \propto \dot{m}$ and the size of the active region $R \propto \dot{m}^{-4 / 3}$ [미]. The magnetic field is assumed to be constant. With an increase of the accretion rate, the X-ray luminosity increases, whereas the optical radiation drops due to the increased synchrotron self-absorption and vice versa (Fig. $\mathbb{W}$, dotted and dashed lines). This makes the spectrum pivoting with spectral hardening during X-ray flares and softening at X-ray dips. Thus, the optical synchrotron luminosity is expected to anti-correlate with the $\mathrm{X}$-rays.

\section{Timing modeling}

We assume that optical radiation is a sum of two components: the synchrotron radiation from the hot flow $s(t)$ and the reprocessed $\mathrm{X}$-ray radiation coming from the accretion disk $d(t)$. According to our findings in Sect. 凤, the synchrotron luminosity drops with increasing X-ray luminosity. We model the behavior as linear anti-correlation: $s(t) \propto-x(t)$. Reprocessed radiation from the disk is a convolution of the X-ray light curve with the disk response function: $d(t) \propto \int_{-\infty}^{t} r\left(t-t^{\prime}\right) x\left(t^{\prime}\right) d t^{\prime}$. 

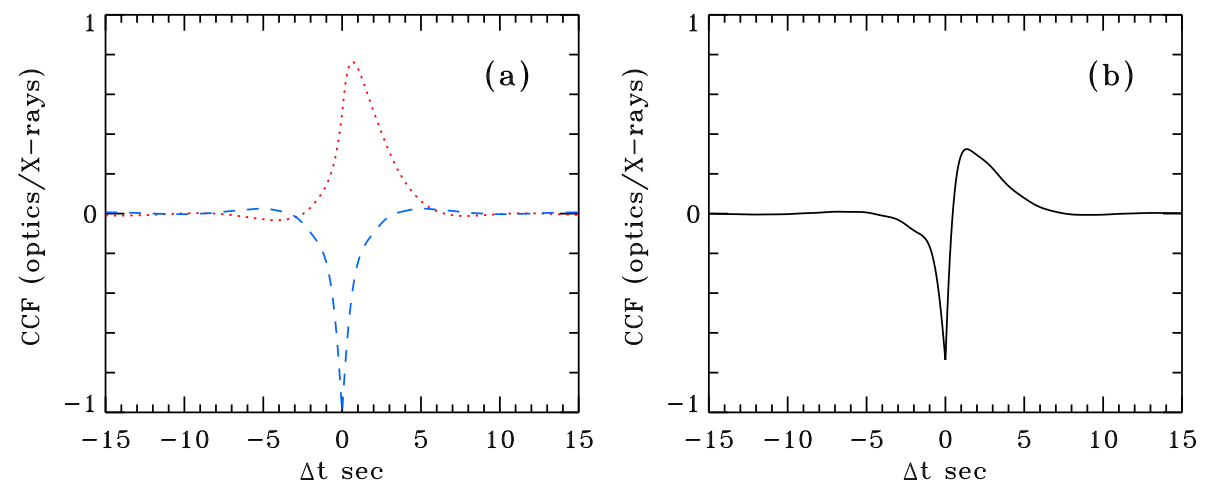

Figure 2: CCFs from the synchrotron (a, dashed), the disk (a, dotted) and their mutual contribution (b).

As an illustration, let us specify a simple exponential response function:

$$
r(t)=\left\{\begin{array}{cl}
\exp \left[-\left(t-\tau_{1}\right) / \tau_{2}\right] / \tau_{2}, & t \geqslant \tau_{1}, \\
0, & t<\tau_{1},
\end{array}\right.
$$

where $\tau_{1}$ corresponds to the delay time of the disk response and $\tau_{2}$ is the response width. The corresponding Fourier image is $R(f)=\exp \left(\mathrm{i} x_{1}\right) /\left(1-\mathrm{i} x_{2}\right)$, where $x_{i}=2 \pi f \tau_{i}$. The total optical light-curve can then be represented as

$$
o(t) \propto-x(t)+r_{\mathrm{ds}} \int_{-\infty}^{t} x\left(t^{\prime}\right) r\left(t-t^{\prime}\right) d t^{\prime},
$$

where we introduced the coefficient $r_{\mathrm{ds}}$, which physically means the ratio of absolute (by amplitude) disk to synchrotron contributions in the variable optical component.

The optical/X-ray CCF also contains two terms: the one arising from synchrotron (it is just an auto-correlation function of the X-rays with a negative sign) and the other one from the disk. Simulated CCFs for separate components and their joint contribution are shown in Fig. ㅁ. The width of the precognition dip depends on the shape of the X-ray power spectral density $P S D_{\mathrm{X}}$. Amplitudes of both negative and positive peaks are reduced, when there are two components in the optics, as they enter the expression (B.2) with different signs and thus partially cancel each other.

To simulate the X-ray light-curve, we define the shape of $\operatorname{PSD}_{\mathrm{X}}(f)$, then calculate the X-ray Fourier transform $X(f)$ with phase being a random number from the interval $[0 ; 2 \pi)$, from which we get $x(t)$. We choose the PSD normalization so that the integral over positive frequencies gives the square of fractional root-mean square (rms) amplitude of the X-ray variability [[3]. Optical PSD is normalized in a similar manner. As an illustration, we choose $\mathrm{rms}_{\mathrm{x}}=1.0$ and $\mathrm{rms}_{\mathrm{o}}=0.3$. If the X-ray PSD is fixed, three parameters control the shape of the CCF and the optical PSD (which we specify here): $r_{\mathrm{ds}}=1.2, \tau_{1}=0.1$ and $\tau_{2}=1.5$. We choose the twice-broken power-law X-ray $\operatorname{PSD}\left(P S D_{\mathrm{X}} \propto f^{0}, f^{-1}\right.$ and $\left.f^{-2}\right)$ with corresponding break frequencies at $0.1 \mathrm{~Hz}$ and $0.5 \mathrm{~Hz}$. This shape is typical for BHBs in their hard state.

Optical Fourier amplitudes are the sum of the synchrotron $(S(f)=-X(f))$ and the disk $\left(D(f)=r_{\mathrm{ds}} X(f) R(f)\right)$ amplitudes:

$$
O(f) \propto X(f)\left[-1+r_{\mathrm{ds}} R(f)\right] .
$$



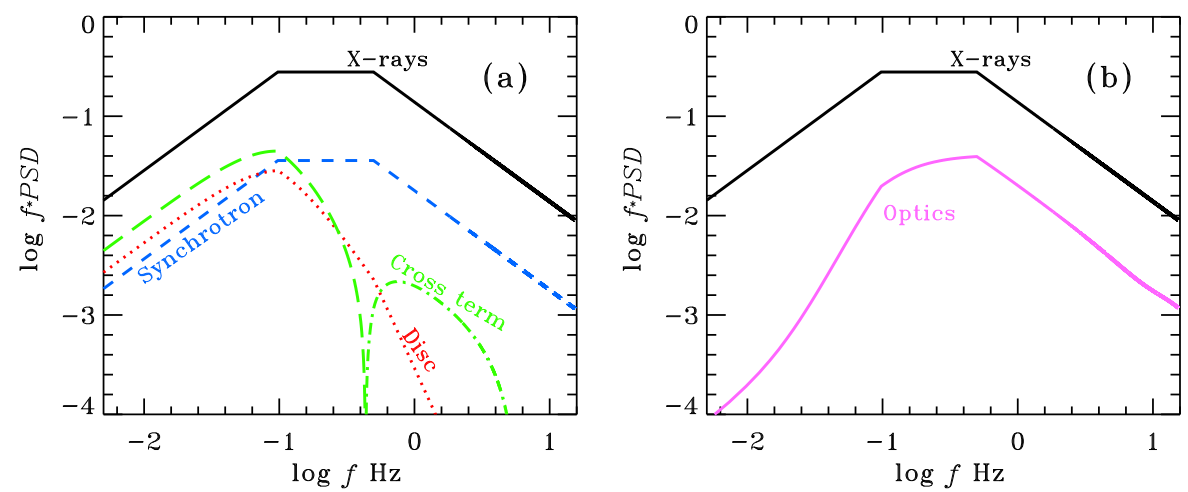

Figure 3: The X-ray PSD (solid) and the optical PSD components (a): synchrotron (dashed), disk (dotted), positive contribution from the cross-term (dot-dashed) and negative contribution from the cross-term (long-dashed). The resulting optical PSD is shown on panel (b).

Optical power spectrum consists of three terms: the synchrotron term, the disk term and a cross term

$$
P S D_{\mathrm{O}} \propto P S D_{\mathrm{X}}\left\{1+r_{\mathrm{ds}}^{2}|R(f)|^{2}-2 r_{\mathrm{ds}} \operatorname{Re}[R(f)]\right\} .
$$

Contributions from different terms and total PSD are shown in Fig. Bla. The first (synchrotron) term has the same shape, as the X-ray PSD. The second term (disk) acts as a low-pass filter. The last PSD (cross-)term changes sign, giving positive contribution to the high-frequency part and negative contribution to the low-frequency part. This makes a bump at frequencies $\sim 0.1-1 \mathrm{~Hz}$ in the optical power spectrum (which was found in analysis of GX339-4, [䧃]). As a consequence of this bump in Fourier space, in the time space the optical ACF becomes narrower. Physically, the high frequencies originate from the interplay of the components, which also results in the fast rise of the CCF at zero lag. The mutual contribution of the two components slightly increases the power at higher frequencies at the same time strongly suppressing lower frequencies.

\section{Summary and discussion}

In this letter, we present a novel model capable of explaining optical/X-ray timing features observed in LMXBs. We argue that the optical radiation is partially produced in the inner parts of the accretion flow by synchrotron mechanism, which also provides seed photons for Comptonization. Additional contribution to the optics comes from reprocessing of the X-ray emission in the outer parts of the cold accretion disk. We show that the presence of the two components can explain the observed shape of the CCF, with the optical precognition dip. For the parameters considered in the present investigations, the shape of the CCFs resemble those found in Swift J1753.5-0127. The model also reproduces another interesting feature observed in the LMXBs, namely, the optical ACF being narrower, than the X-ray one. The explanation of the feature comes from the optical $\mathrm{PSD}$, in which the low-frequency part is suppressed and the bump at frequencies $\sim 0.1-1 \mathrm{~Hz}$ is present, both due to the presence of the cross-term. Physically, the high frequencies originate from the interplay of the two components, which also results in the fast rise of the CCF at zero lag. 


\section{References}

[1] M. Durant et al., SWIFT J1753.5-0127: A Surprising Optical/X-Ray Cross-Correlation Function, ApJ 682 (2008) L45 [arXiv: 0806.2530$]$

[2] M. Durant, P. Gandhi, T. Shahbaz, H. H. Peralta, \& V. S. Dhillon, Multiwavelength spectral and high time resolution observations of SWIFTJ1753.5-0127: new activity?, MNRAS 392 (2009) 309 [arXiv:0810.1141]

[3] M. Durant et al., High time resolution optical/X-ray cross-correlations for $X$-ray binaries: anticorrelations and rapid variability, MNRAS 410 (2011) 2329 [arXiv: 1008 . 4522]

[4] P. Gandhi et al., Rapid optical and X-ray timing observations of GX339-4: multicomponent optical variability in the low/hard state, MNRAS 407 (2010) 2166 [arXiv: 1005 . 4685]

[5] P. Gandhi et al., Rapid optical and X-ray timing observations of GX 339-4: flux correlations at the onset of a low/hard state, MNRAS 390 (2008) L29 [arXiv : 0807 . 1529]

[6] R. I. Hynes et al., The remarkable rapid X-ray, ultraviolet, optical and infrared variability in the black hole XTE J1118+480, MNRAS 345 (2003) 292 [astro-ph / 0306626 ]

[7] G. Kanbach, C. Straubmeier, H. C. Spruit, \& T. Belloni, Correlated fast X-ray and optical variability in the black-hole candidate XTE J1118+480, Nature 414 (2001) 180

[9] J. Malzac, T. Belloni, H. C. Spruit, \& G. Kanbach, The optical and X-ray flickering of XTE J1118+480, A\&A 407 (2003) 335 [astro-ph/ 0306256 ]

[10] J. Malzac \& R. Belmont, The synchrotron boiler and the spectral states of black hole binaries, MNRAS 392 (2009) 570 [arXiv: 0810 . 4458 ]

[11] J. Malzac, A. Merloni, \& A. C. Fabian, Jet-disc coupling through a common energy reservoir in the black hole XTE J1118+480, MNRAS 351 (2004) 253 [astro-ph / 0402674 ]

[12] M. McConnell et al., Observations of Cygnus X-1 COMPTEL during 1991, ApJ 424 (1994) 933

[13] S. Miyamoto \& S. Kitamoto, X-ray time variations from Cygnus X-1 and implications for the accretion process, Nature 342 (1989) 773

[14] C. Motch, M. J. Ricketts, C. G. Page, S. A. Ilovaisky \& C. Chevalier, Simultaneous X-ray/optical observations of GX339-4 during the May 1981 optically bright state, A\&A 119 (1983) 171

[15] J. Poutanen, Accretion disc-corona models and X/ $\gamma$-ray spectra of accreting black holes, in proceedings of Theory of Black Hole Accretion Disks, [ast ro-ph/9805025]

[16] J. Poutanen \& I. Vurm, On the Origin of Spectral States in Accreting Black Holes, ApJ 690 (2009) L97 [arXiv:0807.3073]

[17] A. Różańska \& B. Czerny, Vertical structure of the accreting two-temperature corona and the transition to an ADAF, A\&A $\mathbf{3 6 0}$ (2000) 1170

[18] I. Vurm \& J. Poutanen, Electron thermalization and photon emission from magnetized compact sources, Int. J. Mod. Physics 17 (2008) 1629 [arXiv : 0802 .3680]

[19] I. Vurm \& J. Poutanen, Time-Dependent Modeling of Radiative Processes in Hot Magnetized Plasmas, ApJ 698 (2009) 293 [arXiv: 0807.2540 ]

[20] G. Wardziński \& A. A. Zdziarski, Effects of non-thermal tails in Maxwellian electron distributions on synchrotron and Compton processes, MNRAS 325 (2001) 963 [astro-ph / 0102035 ]

[21] A. A. Zdziarski \& M. Gierliński, Radiative Processes, Spectral States and Variability of Black-Hole Binaries, Prog. Theor. Phys. Suppl. 155 (2004) 99 [astro-ph/ 0403683 ] 